\title{
p53 calls upon CIA (Calcium Induced Apoptosis) to counter
}

\section{stress}

\author{
Sue Haupt ${ }^{1,2}$, Dinesh Raghu ${ }^{1,2,3}$ and Ygal Haupt ${ }^{1,2,3,4 *}$ \\ 1 Tumour Suppression Laboratory, Peter MacCallum Cancer Centre, Melbourne, VIC, Australia \\ 2 Sir Peter MacCallum Department of Oncology, The University of Melbourne, Parkville, VIC, Australia \\ ${ }^{3}$ Department of Pathology, The University of Melbourne, Parkville, VIC, Australia \\ ${ }^{4}$ Department of Biochemistry and Molecular Biology, Monash University, Melbourne, VIC, Australia \\ ${ }^{*}$ Correspondence: ygal.haupt@petermac.org \\ Edited by: \\ Carlotta Giorgi, University of Ferrara, Italy \\ Reviewed by: \\ Francesco Massimo Lasorsa, Consiglio Nazionale delle Ricerche (CNR), Italy
}

Keywords: p53, SERCA, apoptosis, mitochondria, endoplasmic reticulum

\section{A commentary on}

p53 at the endoplasmic reticulum regulates apoptosis in a $\mathrm{Ca}^{2+}$-dependent manner

by Giorgi C, Bonora $M$, Sorrentino $G$, Missiroli S, Poletti F, Suski JM, et al. Proc Natl Acad Sci US A (2015) 112(6):1779-84. doi:10.1073/pnas.1410723112

Intravital imaging reveals p53-dependent cancer cell death induced by phototherapy via calcium signaling

by Giorgi C, Bonora M, Missiroli S, Poletti F, Ramirez FG, Morciano G, et al. Oncotarget (2015) 6(3):1435-45.

The tumor suppressive function of $\mathrm{p} 53$ is a fundamental barrier against cancer development. This is attested by the high frequency of p53 mutations in human cancer, more than any other known gene. Therefore, the mechanisms by which p53 suppresses cancer have attracted much attention. These can be broadly divided into functions that inhibit the growth and propagation of cancer or transformed cells (growth inhibitory activities), and functions that prevent cells from becoming cancerous [reviewed in Ref. (1)]. The mechanisms underlying tumor prevention by p53 are poorly defined and have recently attracted a new wave of interest [reviewed in Ref. (2)]. On the other hand, much more is understood about the mechanisms by which p53 eliminates cancer cells. In response to oncogenic stress, as well as other stress conditions, p53 triggers growth inhibitory functions, including growth arrest, senescence, autophagy, and apoptosis $(1,2) \cdot$ p53 triggers these cellular responses by inducing the expression of a large number of target genes.

While the transcriptional activity of p53 is important for its apoptotic function, an early study provided vital direct indication that a transcriptional deficient p53 mutant is able to induce cell death (3). This report provided the rationale for investigating the transcriptional-independent apoptotic activities of p53. Over the past two decades, the cytoplasmic tumor suppressive functions of p53 have been studied, revealing a role for cytoplasmic p53 in mitochondrial apoptosis and in the suppression of autophagy, which acts as a survival mechanism under metabolic stress conditions [reviewed in Ref. (4)]. It is important to note that both processes are also controlled by nuclear p53: the mitochondrial apoptosis through the powerful induction of Puma, Bax, and Noxa; and autophagy by the induction of genes such as DRAM.

Giorgi and co-workers recently described an important addition to our understanding of the cytoplasmic apoptotic function of $\mathrm{p} 53$. In their studies published in PNAS (5) and Oncotarget (6), they utilized novel calcium imaging tools to provide a new and timely interpretation of how cytoplasmic p53 provokes cell death. They show that in response to stresses, wild type (wt) p53 accumulates at the endoplasmic reticulum-mitochondrial-associated membranes (ER-MAMs), where it interacts with sarco-endoplasmic reticulum $\mathrm{Ca}^{2+}$ ATPase (SERCA) pump to promote calcium overload in the mitochondria. In turn, this drives the loss of membrane potential and culminates in apoptosis (Figure 1). This novel finding provides a vital fragment of clarification for the p53 conundrum regarding its functions independent of its transcriptional activity.

Linking p53, the pivotal orchestrator of cellular stress responses to calcium $\left(\mathrm{Ca}^{2+}\right)$, which is a vital transducer of cell death, ties together two fundamental dictators of apoptosis. Genotoxic damage and oxidative stress are both effective stimuli of p53driven calcium activation. $\mathrm{Ca}^{2+}$ concentration is critical for its functions (including its wider role as a vital secondary messenger in cell signaling), which defines the importance of calcium pumps to generate gradients across membranes. These findings predict that the p53-SERCA connection would not be functional in cancer cells. Consistent with this prediction, SERCA expression is frequently decreased or lost in colon carcinomas and is low in colon and gastric cell lines (7). Similarly, the p53-SERCA function is compromised in breast cancer, where SERCA expression is lost with increased tumor aggressiveness [Ref. (8); reviewed in Ref. (9)]. Importantly, Giorgi et al. (6) showed that the interaction between p53 and SERCA is lost when p53 is mutated. These studies demonstrate the importance of deregulating the p53-SERCA functions during cancer progression either by depleting SERCA or selecting for p53 mutations. It will also be interesting to examine whether the p53 SNP72 impacts on the efficiency of SERCA-mediated apoptosis, where codon 72 Arg was shown to be more prone to mitochondrial apoptosis than codon 72 Pro [reviewed in Ref. (10)]. Significantly, therapeutic targeting of calcium pumps is being investigated [reviewed in Ref. (9)]. For example, SERCA inhibitory agents are considered for the treatment of T-ALL (11). 


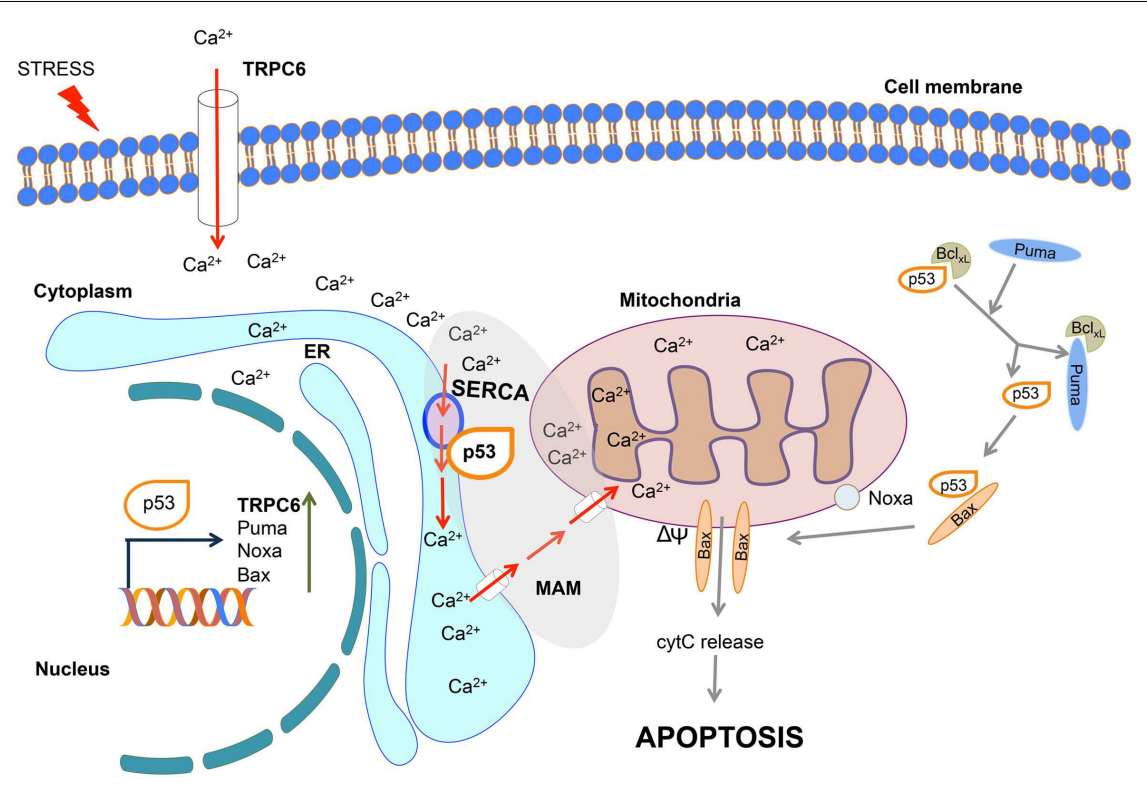

Nuclear role of p53

Cytoplasmic role of p53

FIGURE 1 | Nuclear and cytoplasmic role of p53 in the induction of calcium-induced apoptosis. In response to stress, nuclear p53 activates the transcription of pro-apoptotic genes including TRPC6, Puma, Noxa, and Bax. Cytoplasmic p53 interacts with SERCA at the endoplasmic reticulum (ER)/mitochondrial-associated membrane (MAM) junction, leading to the increase in $\mathrm{Ca}^{2+}$ influx, thereby promoting the loss of membrane potential $(\Delta \Psi)$, release of cytochrome $c$, and induction of apoptosis. The entry of calcium into the cell is further enhanced through TRPC6 channel. Through this combined nuclear and cytoplasmic actions p53 promotes calcium-mediated apoptosis. For simplicity, the other apoptotic functions of p53 are not depicted in this diagram.
The findings by Giorgi et al. argue that in cancers where apoptosis depends on p53SERCA, such therapeutic approaches may be debilitating.

While the studies by Giorgi et al. (5) using a p53 mutant lacking the nuclear localization signal, define a nuclear/transcriptional-independent role for p53 at the ER-mitochondria, p53 has links to calcium beyond this critical function. p53 has recently been demonstrated to transcriptionally activate the calcium channel, transient receptor potential cation channel subfamily C member 6 (TRPC6), in response to the anti-cancer drug derivative of gallium. TRPC6 mediates $\mathrm{Ca}^{2+}$ cellular influx across the plasma membrane (12). Together these fascinating findings implicate calcium to be instrumental in the p53 response both in (1) a transcriptionally dependent manner through elevation of TRPC6 levels to increase cytoplasmic $\mathrm{Ca}^{2+}$ levels and (2) a transcriptionally independent mechanism through its interaction with SERCA driving $\mathrm{Ca}^{2+}$ from the ER into the mitochondria, with a consequent loss of membrane potential.
Thus, p53 is pivotal in both priming and executing cell stress-driven apoptosis in a $\mathrm{Ca}^{2+}$-dependent manner. Once again, as in the case of the mitochondrial apoptotic function of p53 directed through the $\mathrm{BH}$ domain proteins [Puma, Noxa, and Bax reviewed in Ref. (4); Figure 1], here too p53 employs multiple arms to augment the response, and also possibly as a safe guard mechanism. It would be interesting to define the extent of the p53-SERCA apoptotic function in normal cells, during development and whether it is cell type dependent.

\section{ACKNOWLEDGMENTS}

The work in the author lab is supported by NHMRC project grants (1026990, 1026988, 1049179, and 1063389), NHMRC Fellowship to YH (9628426), and by a grant from CCV (APP1085154).

\section{REFERENCES}

1. Selivanova G. Wild type $p 53$ reactivation: from lab bench to clinic. FEBS Lett (2014) 588(16):2628-38. doi:10.1016/j.febslet.2014.03.049

2. Bieging KT, Mello SS, Attardi LD. Unravelling mechanisms of p53-mediated tumour suppres- sion. Nat Rev Cancer (2014) 14(5):359-70. doi:10. 1038/nrc3711

3. Haupt Y, Rowan S, Shaulian E, Vousden KH, Oren M. Induction of apoptosis in HeLa cells by trans-activation-deficient p53. Genes Dev (1995) 9(17):2170-83. doi:10.1101/gad.9.17.2170

4. Comel A, Sorrentino G, Capaci V, Del Sal G. The cytoplasmic side of p53's oncosuppressive activities. FEBS Lett (2014) 588(16):2600-9. doi:10. 1016/j.febslet.2014.04.015

5. Giorgi C, Bonora M, Sorrentino G, Missiroli S, Poletti F, Suski JM, et al. p53 at the endoplasmic reticulum regulates apoptosis in a $\mathrm{Ca} 2+-$ dependent manner. Proc Natl Acad Sci U S A (2015) 112(6):1779-84. doi:10.1073/pnas.1410723112

6. Giorgi C, Bonora M, Missiroli S, Poletti F, Ramirez FG, Morciano G, et al. Intravital imaging reveals p53-dependent cancer cell death induced by phototherapy via calcium signaling. Oncotarget (2015) 6(3):1435-45.

7. Gelebart P, Kovacs T, Brouland JP, Van Gorp R, Grossmann J, Rivard N, et al. Expression of endomembrane calcium pumps in colon and gastric cancer cells. Induction of SERCA3 expression during differentiation. J Biol Chem (2002) 277(29):26310-20. doi:10.1074/jbc.M201747200

8. Papp B, Brouland JP. Altered endoplasmic reticulum calcium pump expression during breast tumorigenesis. Breast Cancer (Auckl) (2011) 5:163-74. doi:10.4137/BCBCR.S7481

9. Monteith GR, Davis FM, Roberts-Thomson SJ. Calcium channels and pumps in cancer: 
changes and consequences. J Biol Chem (2012) 287(38):31666-73. doi:10.1074/jbc.R112.343061

10. Grochola LF, Zeron-Medina J, Meriaux S, Bond GL. Single-nucleotide polymorphisms in the p53 signaling pathway. Cold Spring Harb Perspect Biol (2010) 2(5):a001032. doi:10.1101/ cshperspect.a001032

11. Ilagan MX, Kopan R. Selective blockade of transport via SERCA inhibition: the answer for oncogenic forms of Notch? Cancer Cell (2013) 23(3):267-9. doi:10.1016/j.ccr.2013.02.020

12. Madan E, Gogna R, Keppler B, Pati U. p53 increases intra-cellular calcium release by transcriptional regulation of calcium channel
TRPC6 in GaQ3-treated cancer cells. PLoS One (2013) 8(8):e71016. doi:10.1371/journal.pone. 0071016

Conflict of Interest Statement: The authors declare that the research was conducted in the absence of any commercial or financial relationships that could be construed as a potential conflict of interest.

Received: 16 February 2015; paper pending published: 21 February 2015; accepted: 23 February 2015; published online: 10 March 2015.
Citation: Haupt S, Raghu D and Haupt Y (2015) p53 calls upon CIA (Calcium Induced Apoptosis) to counter stress. Front. Oncol. 5:57. doi: 10.3389/fonc.2015.00057 This article was submitted to Molecular and Cellular Oncology, a section of the journal Frontiers in Oncology. Copyright (C) 2015 Haupt, Raghu and Haupt. This is an open-access article distributed under the terms of the Creative Commons Attribution License (CC BY). The use, distribution or reproduction in other forums is permitted, provided the original author(s) or licensor are credited and that the original publication in this journal is cited, in accordance with accepted academic practice. No use, distribution or reproduction is permitted which does not comply with these terms. 